Article

\title{
An Injectable, Dual Responsive, and Self-Healing Hydrogel Based on Oxidized Sodium Alginate and Hydrazide-Modified Poly(ethyleneglycol)
}

\author{
Lei Wang ${ }^{1}$, Wanfu Zhou ${ }^{2}$, Qingguo Wang ${ }^{2}$, Chao $\mathrm{Xu}^{1}{ }^{1}$, Quan Tang ${ }^{1}$ and Haiyang Yang ${ }^{1, *}$ \\ 1 CAS Key Laboratory of Soft Matter Chemistry, School of Chemistry and Materials Science, \\ University of Science and Technology of China, Hefei 230026, China; wl1991@mail.ustc.edu.cn (L.W.); \\ xc94@mail.ustc.edu.cn (C.X.); tangq@mail.ustc.edu.cn (Q.T.) \\ 2 Oil Production Technology Institute, Daqing Oilfield Company Ltd., Daqing 163453, China; \\ zhouwf@petrochina.com.cn (W.Z.);wqg@petrochina.com.cn (Q.W.) \\ * Correspondence: yhy@ustc.edu.cn; Tel.: +86-0551-6360-7549
}

Received: 6 February 2018; Accepted: 28 February 2018; Published: 1 March 2018

\begin{abstract}
Oxidized sodium alginate is a handily modifiable polysaccharide owing to the pendant aldehyde groups which can form dynamic covalent bonds with amines, acylhydrazines, etc., providing oxidized sodium alginate-based hydrogels with stimuli-responsive properties. However, due to the stiffness and, in particular, the hydrophobicity of sodium alginate dialdehyde at low $\mathrm{pH}$, the mechanical performance and $\mathrm{pH}$ stimuli responsiveness of oxidized sodium alginate-based hydrogels are still strictly limited. Herein, we report a new strategy to build an injectable, dual responsive, and self-healing hydrogel based on oxidized sodium alginate and hydrazide-modified poly(ethyleneglycol) (PEG). The hydrazide-modified PEG, referred to as PEG-DTP, acts as a macromolecule crosslinker. We found that the presence of PEG-DTP reduces the hydrophobicity of oxidized sodium alginate at low $\mathrm{pH}$ so effectively that even a $\mathrm{pH}$-induced reversible sol-gel transitions can be realized. Meanwhile, the disulfide bonds in PEG-DTP endows the hydrogel with the other reversible sol-gel transitions by redox stimuli. In particular, due to the softness of PEG-DTP chains, mechanical performance was also enhanced significantly. Our results indicate we can easily integrate multi-stimuli responsiveness, injectability, and self-healing behavior together into an oxidized sodium alginate-based hydrogel merely by mixing an oxidized sodium alginate solution with PEG-DTP solution in certain proportions.
\end{abstract}

Keywords: oxidized sodium alginate; dynamic covalent bonds; stimuli-responsive; sol-gel transitions; injectable hydrogel; self-healing

\section{Introduction}

With high water content and their structural similarity to natural extracellular matrix (ECM), hydrogels have be widely studied and applied in the fields of drug delivery [1], tissue engineering [2], and wound healing [3]. To serve as biomaterials, especially as the carriers [4] of drugs or cells scaffold [5], hydrogels should incorporate the properties of biocompatibility, injectability, and self-healing. Meanwhile, for better practical applications, some recently developed hydrogels are endowed with attractive external stimuli-responsiveness. The injectability facilitates the hydrogel to incorporate therapeutic drugs and cells via simple mixing and a minimally invasive [6] surgical procedure, and with a decreased risk of implant migration [7]. Self-healing hydrogels are soft materials which can quickly repair the damaged network. In case of unexpected breakage of the gel matrix and the subsequent inflammation risk caused by the fragmented gels, the ability to self-heal is important for hydrogels applied in vivo [8]. Besides, some dynamic covalent interactions or physical interactions are 
utilized to prepare hydrogels with stimuli-responsive properties [9-11]. By the triggers of $\mathrm{pH}[12,13]$, reducing agents [14], temperature [15], or light [16], hydrogels can be degraded and the incorporated drugs can be released. For example, Zhang et al. [17] prepared multi-responsive polymer hydrogels based on the dynamic boronate ester and disulfide bonds. In response to $\mathrm{pH}$, glucose, and redox, the hydrogel exhibited gel-sol-gel transitions, showing the potential use as drug carrier. Therefore, if the hydrogel is simultaneously injectable, self-healing [18-20], and stimuli-responsive, it would be of great value.

As a natural polymer, sodium alginate is biocompatible and in an oxidizing environment part of its constitutional units can be oxidized to aldehyde groups through ring opening reactions [21]. The sodium alginate dialdehyde (ADA) with numerous aldehyde groups can react with a number of groups, such as amines, to form dynamic covalent bonds [22]. The sodium alginate-based hydrogels, therefore, can be endowed easily with stimuli-responsive properties. As an important crosslinker, 3,3'-dithiobis(propionohydrazide) (DTP) [23] with hydrazide and disulfide functional groups can react with aldehyde groups by Schiff base reactions to from acylhydrazone crosslinks. Since the Schiff base reaction can be quickly conducted at mild conditions, hydrogels are able to form in situ after mixing and injecting the precursor solutions. For example, injectable $\mathrm{pH}$-responsive hollow particle gels utilizing DTP via Schiff base reaction were prepared by Brian R. Saunders [24]. Besides, the dynamic acylhydrazone bonds and disulfide linkages endow the hydrogels with self-healing and stimuli-responsive properties. However, due to the stiffness of the oxidized sodium alginate chain, the hydrogels based on oxidized sodium alginate crosslinked by DTP are brittle. In particular, the oxidized sodium alginate itself will dehydrate in acidic conditions, showing a poor $\mathrm{pH}$ responsiveness.

Herein, we synthesized sodium alginate dialdehyde (ADA) and 3,3'-dithiobis (propionohydrazide) (DTP) modified PEG (PEG-DTP) to prepare an injectable, stimuli-responsive, and self-healing hydrogel. PEG-DTP/ADA hydrogel was prepared via the Schiff base reaction between PEG-DTP and ADA. The soft macromolecular crosslinker (PEG-DTP) improved the flexibility of the network effectively and the mechanical performance of PEG-DTP hydrogel was obviously improved. Besides, the more hydrophilic crosslinker PEG-DTP obviously enhanced the hydrophilicity of the hydrogel network, contributing to a better $\mathrm{pH}$ responsiveness of the hydrogel. Since the gelation time can be tuned by adjusting $\mathrm{pH}$, the PEG-DTP/ADA hydrogel showed good injectability. The dynamic acylhydrazone bonds which can self-regenerate after breaking provide the hydrogel with a $100 \%$ self-healing ability at room temperature. Moreover, the $\mathrm{pH}$-sensitive acylhydrazone bonds and redox-sensitive disulfide linkages endowed the PEG-DTP/ADA hydrogel with dual responsive properties. Accordingly, the degradation of the hydrogel was controllable by varying $\mathrm{pH}$ or reductant concentrations. The hydrogel was applied to control release of rhodamine B and distinct 1,4-dithiothreitol (DTT) controlled release behaviors were observed. Because of the outstanding biocompatibility of sodium alginate and PEG, the PEG-DTP/ADA hydrogel was non-cytotoxic, as testified by in vitro cytotoxicity evaluation.

\section{Results and Discussion}

\subsection{Preparation and Rheological Characterization of PEG-DTP/ADA Hydrogel}

Aldehyde sodium alginate (ADA) was the oxidative product of sodium alginate, oxidized by sodium periodate (Figure S1). The oxidization of ALG to ADA was verified by the FT-IR spectra of sodium alginate (ALG) and oxidized sodium alginate (ADA) (Figure S2), Due to the absorption peak of ADA, a new absorption peak corresponding to the characteristic band of a carbonyl group of ADA appeared at $1726 \mathrm{~cm}^{-1}$, showing the successful preparation of ADA. The oxidation degree of sodium alginate was about $35 \%$, as determined by the hydroxylamine hydrochloride titration method [25]. PEG-DTP was obtained by the amidation between DTP and PEG-diacid (Figure S3). The structures of PEG-diacid and PEG-DTP were characterized by ${ }^{1} \mathrm{H}-\mathrm{NMR}$. As shown in Figure S3, the peak $\mathrm{b}$ (4.45 ppm) on the spectrum of PEG-diacid originated from the methene near the carboxy group, 
which demonstrated that poly(ethyleneglycol) was oxidized to PEG-diacid. In Figure S4, peak "c" (2.88 ppm, methene near the disulfide bond) and " $\mathrm{d}$ " (2.51 ppm, methene near the hydrazide bond) on the ${ }^{1} \mathrm{H}-\mathrm{NMR}$ spectrum indicated that PEG-diacid and DTP had reacted to form PEG-DTP.

As we all know, The Schiff base reaction can be conducted at mild conditions (room temperature, wide $\mathrm{pH}$ range) to form hydrogel. As depicted in Figure 1, the hydrogel was facilely prepared by mixing the aqueous ADA solution (20 wt \%) with the aqueous PEG-DTP solution ( $20 \mathrm{wt} \%)$. The Schiff base reaction between ADA and PEG-DTP is quite fast, such that the dynamic network was quickly formed and the gel was obtained in several minutes (Figure S5). A series of PEG-DTP/ADA hydrogels with different mass ratios of PEG-DTP/ADA and different solid content were prepared, noted as $\mathrm{Gel}_{\mathrm{X}-\mathrm{Y}}$ (Table S1). Herein, "Y" means the mass ratio of two components (PEG-DTP:ADA = 1, 2, 3, 4) and " $x$ " means different solid content of hydrogels (15 wt \% and $20 \mathrm{wt} \%)$.

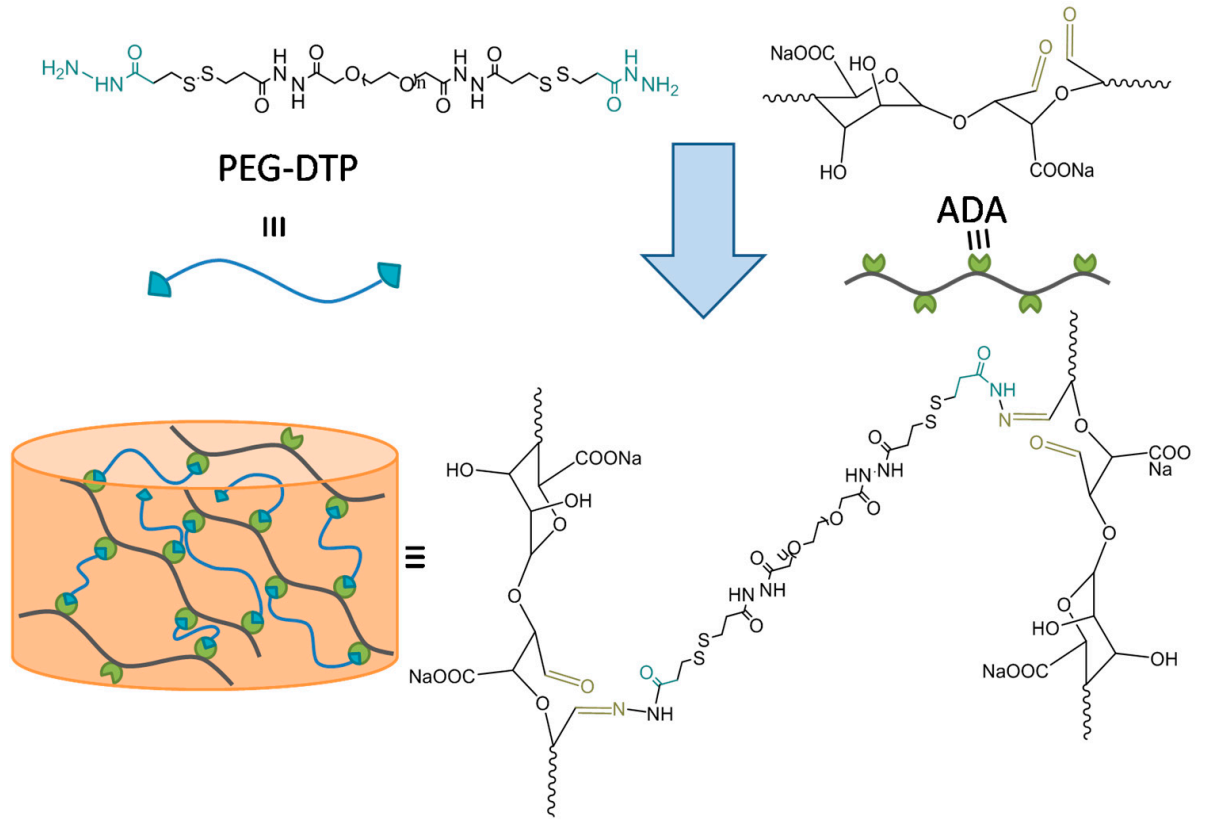

Figure 1. Schematic diagram of the preparation of the PEG-DTP/ADA hydrogel.

\subsection{Mechanical Properties of ADA/PEG-DTP Hydrogels}

The mechanical properties of the prepared hydrogels were measured by rheometer (TA Instruments, AR-G2). As shown in Figure 2a, the elastic modulus $\mathrm{G}^{\prime}$ of the hydrogel was larger than the loss modulus $G^{\prime \prime}$ in the whole frequency range, demonstrating the sample was always in the gel state. As solid content increased, the elastic modulus $\mathrm{G}^{\prime}$ of the $\mathrm{Gel}_{20}-3$ hydrogel was higher than the $\mathrm{Gel}_{15}-3$ hydrogel, although they had similar loss modulus G" $(20 \mathrm{~Pa})$. The better elasticity of $\mathrm{Gel}_{20}-3$ indicated that more robust crosslinking networks were formed in the high solid content hydrogel, resulting in better mechanical performance. When varying the mass ratio of the two components of the hydrogels (PEG-DTP:ADA = 1, 2, 3, 4), the $\mathrm{G}^{\prime}$ of $\mathrm{Gel}_{20}-3$ was higher than other hydrogels, while the $G^{\prime \prime}$ of these hydrogels were similar (Figure $2 b$ ). This revealed that the mass ratio of PEG-DTP:ADA $=3: 1$ was optimum to form a strong ADA/PEG-DTP hydrogel. With better mechanical properties, $\mathrm{Gel}_{20}-3$ was selected to test the other properties of the ADA/PEG-DTP hydrogels, as displayed below.

It is noted that the PEG-DTP/ADA hydrogel showed better mechanical properties than the DTP / ADA hydrogel crosslinked by DTP (Figure 3). The PEG-DTP/ADA hydrogel was able to sustain a compressive strain of over $60 \%$, whereas the DTP/ADA hydrogel was broken at a compressive strain of 50\%. In the DTP/ADA hydrogel, simply crosslinking the stiff ADA molecule with the small molecule crosslinker DTP generated a quite rigid network and the resulting hydrogel was brittle. By contrast, the soft macromolecular crosslinker PEG-DTP could effectively improve the flexibility of the network. Thus, the mechanical performance of the PEG-DTP hydrogel was obviously improved. 


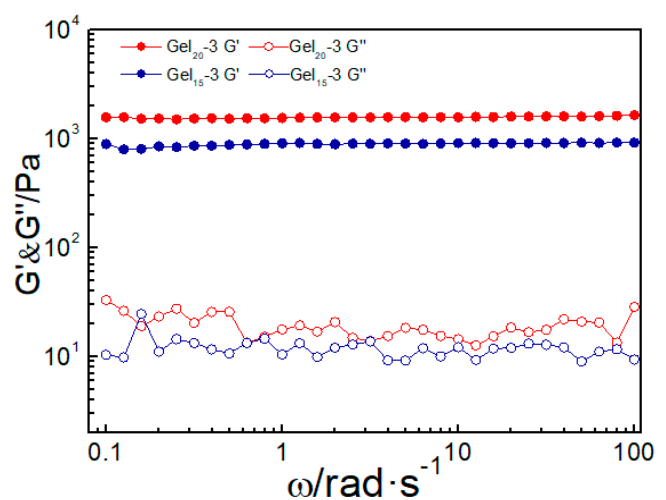

(a)

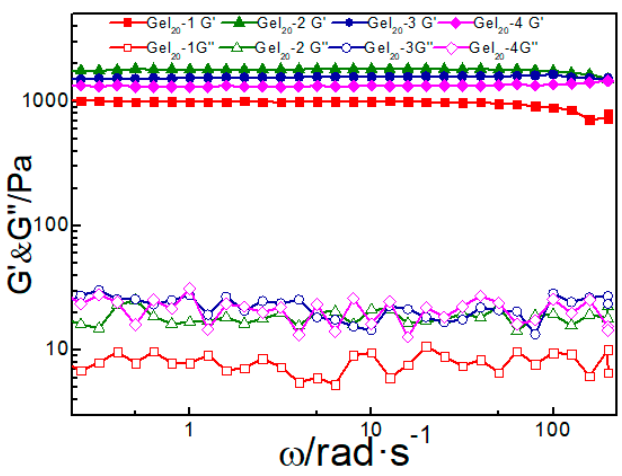

(b)

Figure 2. (a) Frequency sweep measurements of hydrogels $\mathrm{Gel}_{15}-3$ and $\mathrm{Gel}_{20}-3$; (b) Storage moduli $\left(\mathrm{G}^{\prime}\right)$ and loss moduli $\left(\mathrm{G}^{\prime \prime}\right)$ of hydrogels $\mathrm{Gel}_{20}-1, \mathrm{Gel}_{20}-2, \mathrm{Gel}_{20}-3$, and $\mathrm{Gel}_{20}-4$ as a function of angular frequency.

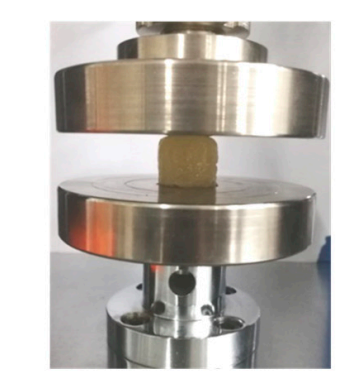

PEG-DTP/ADA hydrogel

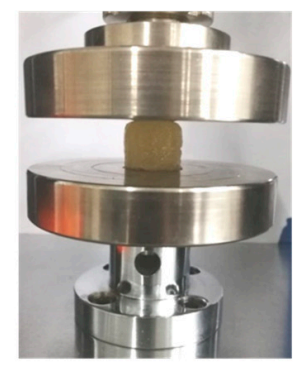

DTP/ADA hydrogel

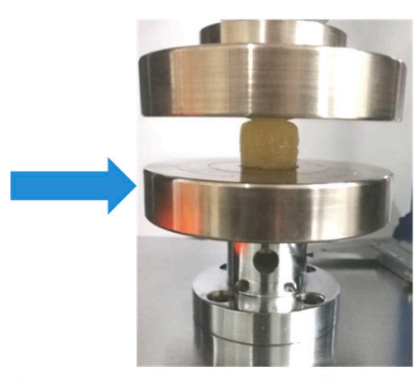

$60 \%$ compression ratio

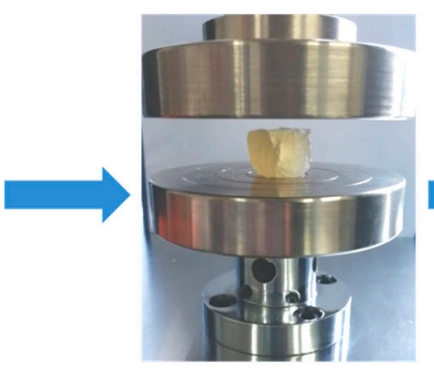

$50 \%$ compression ratio

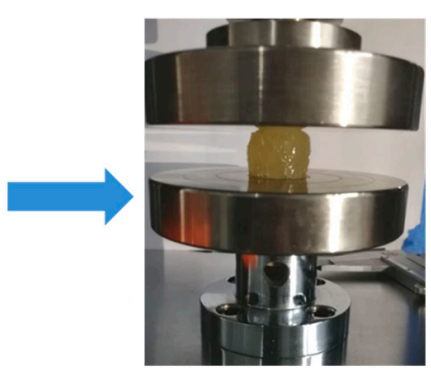

$70 \%$ compression ratio

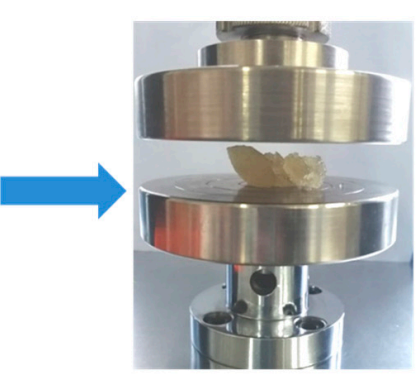

$60 \%$ compression ratio

Figure 3. Compression performance of the PEG-DTP/ADA hydrogel and the DTP/ADA hydrogel.

\subsection{Self-Healing and Injectability of PEG-DTP/ADA Hydrogels}

As mentioned before, to quickly repair a damaged gel matrix, the ability to self-heal is important for the practical application of hydrogels, especially for injectable hydrogels. Before testing the self-healing ability, we conducted a strain sweep measurement on $\mathrm{Gel}_{20}-3$ to test the maximum shear strain the hydrogel could withstand. As shown in Figure $4 a$, the storage moduli $\mathrm{G}^{\prime}$ and the loss moduli $G^{\prime \prime}$ of the hydrogel did not change under the strain $\gamma<60 \%$, showing that the sample could bear a large shear strain. With the strain increased, $G^{\prime}$ dropped while $G^{\prime \prime}$ dramatically raised and the curve had a crossover at strain $\gamma=700 \%$. This indicated the network of hydrogel was totally broken and converted into sol state at strain $\gamma=700 \%$. Then, in order to test the self-healing ability of the hydrogel, repeated dynamic strain step tests $(\gamma=1 \%$ or $\gamma=1200 \%$ ) were carried out (Figure $4 \mathrm{~b}$ ). When the strain increased to $1200 \%$, the hydrogel network was disrupted as the storage moduli $\mathrm{G}^{\prime}$ was only $200 \mathrm{~Pa}$ and the loss moduli G" increased to $400 \mathrm{~Pa}$. After decreasing the strain back to $1 \%$, the $\mathrm{G}^{\prime}$ and $\mathrm{G}^{\prime \prime}$ values of the sample almost recovered to initial values, implying the hydrogel network was able to completely restore itself after it was broken. The tests were repeated three times, and in each cycle, $G^{\prime}$ and $G^{\prime \prime}$ of 
the sample could recover their initial values, further illustrating the $100 \%$ and repeatable self-healing functionality of the PEG-DTP/ADA hydrogels. Besides, Figure 4c also visually demonstrated that the hydrogels had a good self-healing ability at room temperature. The hydrogels were dyed with different colors and cut into two halves. When put them together, the two parts self-healed into an integral hydrogel after $12 \mathrm{~h}$.

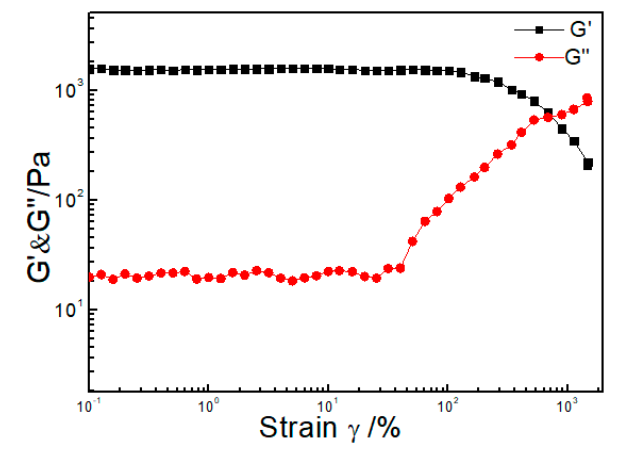

(a)

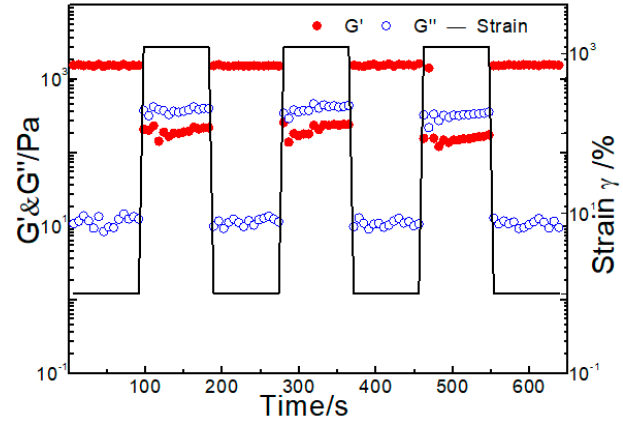

(b)

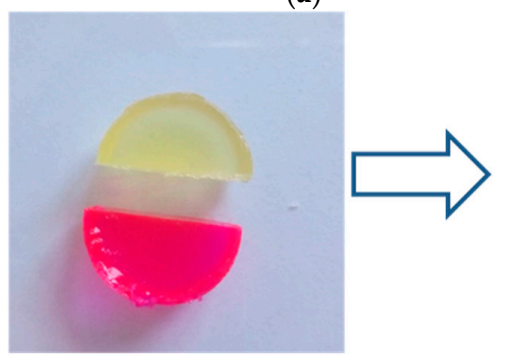

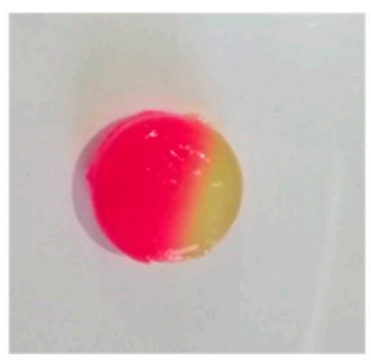

(c)

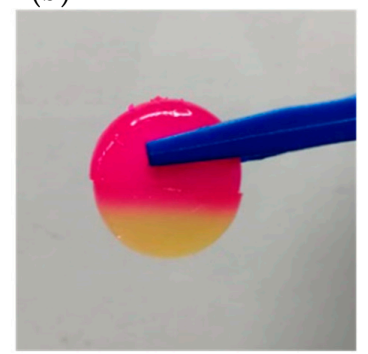

Figure 4. (a) Strain sweep measurements of hydrogel $\mathrm{Gel}_{20}-3$; (b) Repeated dynamic strain step tests (1\% or $1200 \%)$; (c) Images of the self-healing process of two individual gel fragments.

Since the Schiff base reaction between PEG-DTP and ADA can quickly establish chemical crosslinks to form the hydrogel network (Figure 1), the injectability of the PEG-DTP/ADA hydrogel was shown in Figure 5. ADA solution and PEG-DTP solution were in the liquid state when squeezed out (Figure 5a,b). The ADA solution and the PEG-DTP solution were mixed together in a syringe and the gel was formed in situ when squeezed out. For better injectability, the gelation time of the injectable hydrogel should be controllable. The gelation time of the ADA/PEG-DTP hydrogels can be easily controlled due to the $\mathrm{pH}$ sensitivity of the Schiff base reaction. As depicted in Table S2, gelation time measured by vial inversion test [26] at different $\mathrm{pH}$ values $(3.0,5.0,7.0)$ were $10 \mathrm{~s}, 60 \mathrm{~s}$, and $1.5 \mathrm{~h}$. Namely, the gelation time of the hydrogel can be tuned over a wide range by changing the $\mathrm{pH}$. Figure $5 \mathrm{~d}$ shows that the PEG-DTP/ADA hydrogels can be squeezed as bio-ink to write the word "USTC". Together with the tunable gelation time, the PEG-DTP/ADA hydrogel is also a promising candidate as a $3 \mathrm{D}$ printing hydrogel.

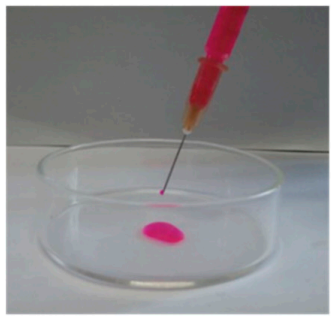

(a)

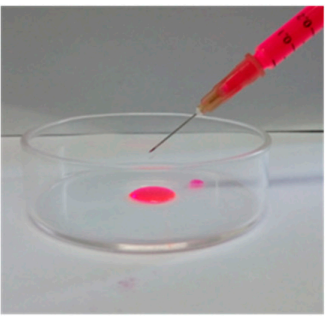

(b)

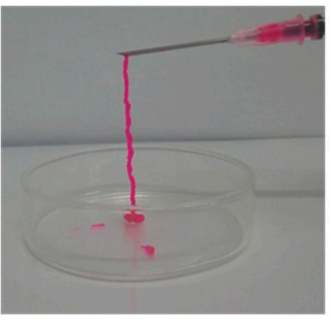

(c)

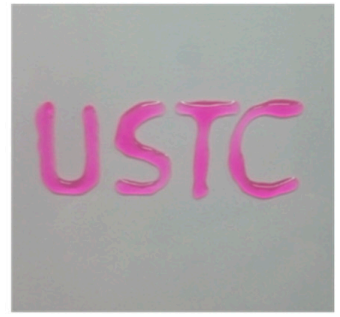

(d)

Figure 5. Injection of ADA solution (a) and PEG-DTP solution (b), injectability (c), and printing ability (d) of hydrogel samples. (Both compositions were dyed by rhodamine B). 


\subsection{Dual Responsive Properties of PEG-DTP/ADA Hydrogels}

The reaction between the $-\mathrm{NH}_{2}$ groups of PEG-DTP and the - $\mathrm{CHO}$ groups of ADA formed the $\mathrm{pH}$-sensitive acylhydrazone bonds, and the disulfide linkage in PEG-DTP were responsive to reductants (DTT or GSH). Thus, the dual responsive properties were displayed in our PEG-DTP/ADA hydrogel system. Acylhydrazone bonds could break at low $\mathrm{pH}$ and reform at high $\mathrm{pH}$, while disulfide linkages could break in reducing environments and reform in oxidizing environments, thus the hydrogel exhibited dual sol-gel transitions. Vial inversion tests [26] were used to monitor the gel-sol-gel transition. As shown in Figure 6, the hydrogel turned into the sol-gel state by adding hydrochloric acid solution to turn the $\mathrm{pH}$ to 1 . Then, when triethylamine was added to change the $\mathrm{pH}$ to 7 , the hydrogel was reformed again. Furthermore, the sol-gel transition was also observed by the triggering of redox stimuli (DTT $\left./ \mathrm{H}_{2} \mathrm{O}_{2}\right)$. The hydrogel turned into the sol-gel state when adding the reductant, DTT, and recovered the hydrogel network after adding the oxidant, $\mathrm{H}_{2} \mathrm{O}_{2}$ (Figure 6). The dual sol-gel transitions indicated that the PEG-DTP/ADA hydrogel has excellent stimuli-responsiveness.

In contrast, the DTP / ADA hydrogel did not show the $\mathrm{pH}$ triggered sol-gel transition (Figure 7). The hydrogel was dehydrated in acidic solutions, which was attributed to the decreased hydrophilicity of the hydrogel network resulting from the protonation of carboxyl groups in the ADA segments. The replacement by DTP in the more hydrophilic crosslinker PEG-DTP obviously improved the hydrophilicity and flexibility of the hydrogel network, contributing to the outstanding $\mathrm{pH}$ responsiveness of the hydrogel.

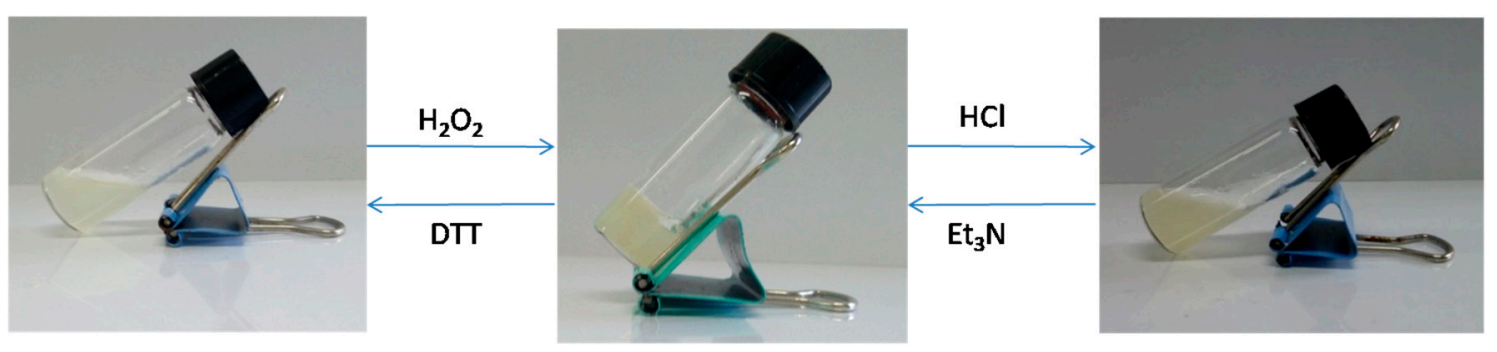

Figure 6. Sol-gel transitions of PEG-DTP/ADA hydrogels in response to $\mathrm{pH}$ or redox triggers.
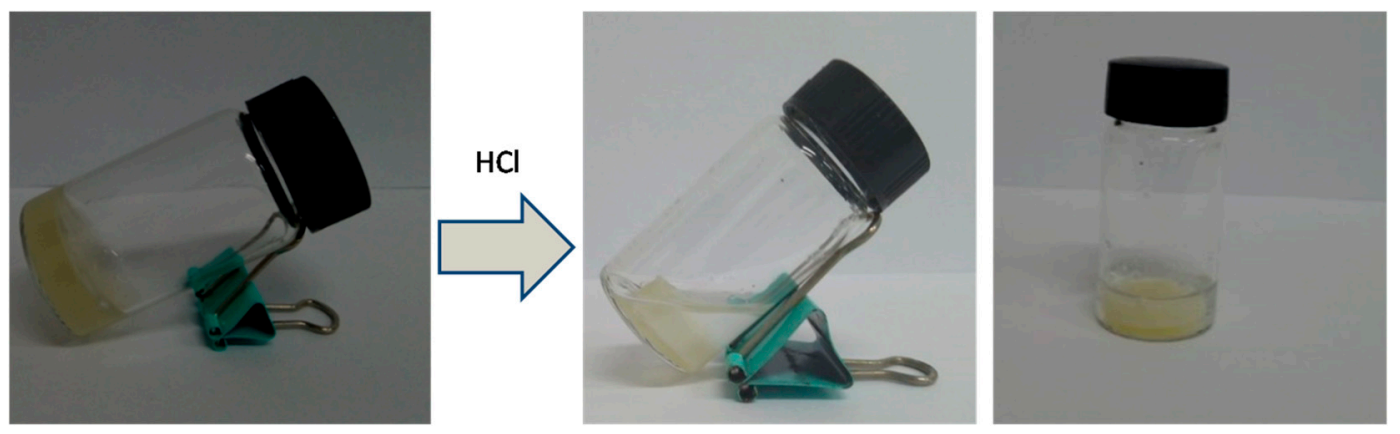

Figure 7. Dehydration of DTP/ADA hydrogel at $\mathrm{pH}=1$.

\subsection{Degradation and Drug Release Ability of PEG-DTP/ADA Hydrogels}

For injectable hydrogels, controllable degradability is of high value for drug release. Due to the stimuli responsiveness, the PEG-DTP/ADA hydrogel exhibited intriguing $\mathrm{pH} /$ redox triggered degradation. Reductants, such as DTT and glutathione (GSH), could break the disulfide crosslinking and hasten the degradation of the hydrogel network. As shown in Figure 8a, the weight loss of the PEG-DTP / ADA hydrogel $\left(\mathrm{Gel}_{20}-3\right)$ was only $20 \mathrm{wt} \%$ in 3 days in $\mathrm{pH} 7.4$ PBS solution. While immersed in DTT solutions for $26 \mathrm{~h}$, the hydrogel showed almost $90 \%$ weight loss in $10 \mathrm{mM}$ DTT solution and $63 \%$ weight loss in $1 \mathrm{mM}$ DTT solution. The obviously different and accelerated degradation rate 
verified that the hydrogel's degradation ratio can be easily controlled by varying the concentration of DTT. Besides, since acylhydrazone bonds were cleavable under acidic conditions, the degradation of PEG-DTP/ADA hydrogel could be triggered by varying $\mathrm{pH}$. As shown in Figure $8 \mathrm{a}$, the hydrogel showed a higher degradation rate at $\mathrm{pH}=5$, compared to $\mathrm{pH}=7.4$. Therefore, the degradability of the ADA/PEG-DTP hydrogel can be easily controlled by adjusting the DTT concentration or $\mathrm{pH}$.

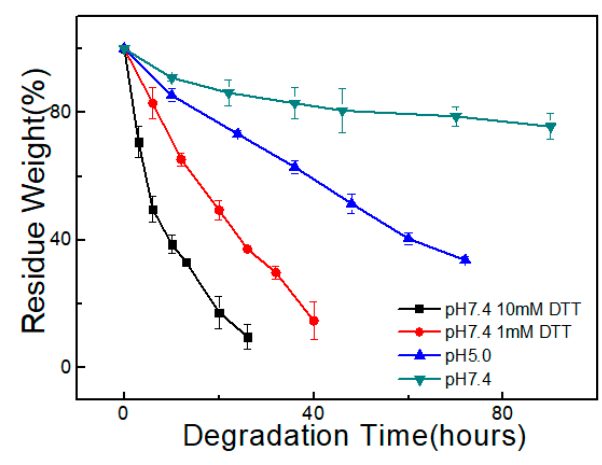

(a)

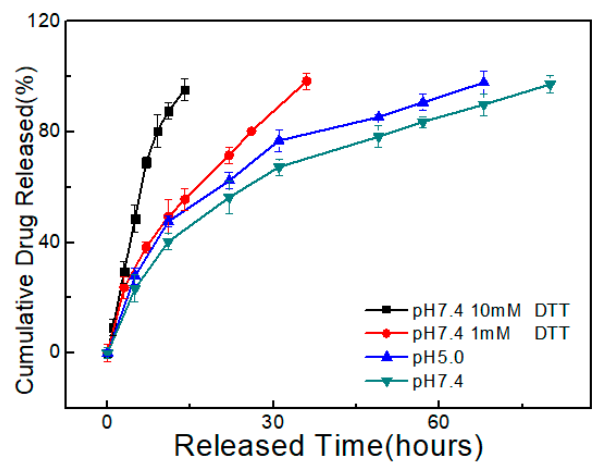

(b)

Figure 8. (a) Degradation and (b) release of a model drug (rhodamine B) by the hydrogels.

With the controllable degradation of the hydrogel network, the drug release rate of a PEG-DTP / ADA hydrogel can be easily controlled. To study the drug release ability of the hydrogel, we used rhodamine $\mathrm{B}$ as the model drug. The in vitro drug release behavior tests were carried out in weakly acidic and/or reductive conditions. As shown in Figure 8b, rhodamine B released nearly $100 \%$ in $12 \mathrm{~h}$ in $10 \mathrm{mM}$ DTT solution, while it took nearly $40 \mathrm{~h}$ to release $100 \%$ in $1 \mathrm{mM}$ DTT solution. On the contrary, when in the $\mathrm{pH} 7.4 \mathrm{PBS}$ solution, it took $80 \mathrm{~h}$ to completely release the drug. Similarly, in $\mathrm{pH}$ 5.0 solution, the rhodamine B released more quickly than in $\mathrm{pH} 7.4$ solution. In view of the biologically relevant microenvironments such as tumor sites, the controllable release of drug under weakly acidic and reductive conditions makes the hydrogel a promising drug carrier.

\subsection{In Vitro Cytotoxicity Evaluation}

As it is well known that poly(ethyleneglycol) and sodium alginate are biocompatible polymers and are widely used in hydrogel preparation, the PEG-DTP/ADA hydrogels are supposed to be biocompatible. A549 cells were utilized to study the cell cytotoxicity of the hydrogel, and were analyzed by MTT assay [27]. The polymer solutions (PEG, PEG-DTP, and ADA) and hydrogel were used to test cell cytotoxicity. As shown in Figure 9, the A549 cells of PEG-DTP solution and ADA solution showed good viability (>90\%) after incubation with the solution for $24 \mathrm{~h}$. The results indicated that PEG, PEG-DTP, ADA, and hydrogel had good biocompatibility, confirming that the hydrogel is a good candidate for use as a drug carrier.

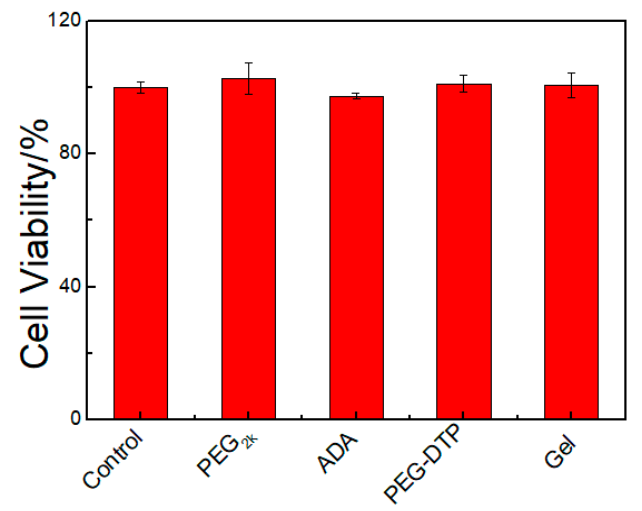

Figure 9. Cell (A549) viability upon exposure to $\mathrm{PEG}_{2 \mathrm{k}}$, ADA, PEG-DPT, and hydrogel. 


\section{Materials and Methods}

\subsection{Materials}

1,4-Dithiothreitol (DTT), dicyclohexylcarbodiimide (DCC), and N-hydroxysuccinimide (NHS) were purchased from Aladdin Reagents (Shanghai, China). 3,3'-Dithiobis (propionohydrazide) (DTP) was purchased from J\&K Scientific (Shanghai, China). Sodium alginate (ALG), sodium periodate, methanol, ethanol, ethylene glycol, poly(ethyleneglycol) (PEG, Mn = 2000), phenolphthalein, concentrated $\mathrm{HCl}$ solution, dimethyl sulfoxide (DMSO), triethylamine $\left(\mathrm{Et}_{3} \mathrm{~N}\right)$, and sodium hydroxide were obtained from Sinopharm Reagents (Shanghai, China) and were used as received without further purification. Murine A549 cells were purchased from SIBCB (Shanghai Institute of Cell Biology, Shanghai, China). Cell culture lysis buffer and MTT salt (3-(4,5-dimethylthiazol-2-yl)-2,5-diphenyltetrazolium bromide) and FBS (fetal bovine serum), trypsin, PBS (phosphate buffered saline), and DMEM (Dulbecco's modified Eagle medium) were purchased from GIBCO (Beyotime Institute of Biotechnology, Shanghai, China).

\subsection{Synthesis of Sodium Alginate Dialdehyde (ADA)}

Oxidized sodium alginate (ADA) was synthesized according to previously reported literature [28]. The specific steps are as follows (Figure S1). First, $10 \mathrm{~g}$ sodium alginate (ALG) was dissolved in $500 \mathrm{~mL}$ deionized water in $1 \mathrm{~L}$ round bottom flask, and then $5.50 \mathrm{~g}$ sodium periodate was dissolved in $100 \mathrm{~mL}$ deionized water. The two solutions were mixed together and stirred for another $6 \mathrm{~h}$ in a dark environment at room temperature. Ethylene glycol, equimolar to sodium periodate, was added and stirred for an hour to quench the reaction. The product mixture was precipitated with excess ethanol. Then, the oxidized product of sodium alginate (ADA) was filtrated and dialyzed (MWCO $=8000 ~ 10,000$ ) for two days. Finally, pure ADA was obtained by freeze-drying.

\subsection{Synthesis of Hydrazide-Terminated Poly(ethyleneglycol) (PEG-DTP)}

3,3'-Dithiobis(propionohydrazide) (DTP) functionalized poly(ethyleneglycol) (PEG-DTP) (Figure S1) was prepared by the amidation reaction [10] between PEG-diacid and 3,3'-dithiobis (propionohydrazide). First, PEG-diacid was synthesized according to a similar procedure of the previous report [29]. Twenty grams of $\mathrm{PEG}_{2 \mathrm{k}}$ was dissolved into $200 \mathrm{~mL}$ acetone in a $500 \mathrm{~mL}$ round bottom flask. Then, the solution was heated to $40{ }^{\circ} \mathrm{C}$ to obtain a clear solution and then it was cooled to room temperature. Next, $10 \mathrm{~mL}$ Jone's reagent $(1.25 \mathrm{M})$ was added dropwise and the reaction mixture was kept at room temperature. The reaction mixture was stirred overnight $(24 \mathrm{~h})$. Then, the reaction was quenched by $6.5 \mathrm{~mL}$ (equimolar to Jone's reagent) isopropyl alcohol. After evaporating off the acetone, the crude product was dissolved by $150 \mathrm{~mL}$ deionized water. The product was extract by dichloromethane $(30 \mathrm{~mL} \times 3)$. Finally, PEG-diacid was obtained after solvent evaporation.

To prepare PEG-DTP, 8 g 3,3'-dithiobis(propionohydrazide) (DTP), $1.16 \mathrm{~g}$ DCC, and $0.65 \mathrm{~g}$ NHS were dissolved into $80 \mathrm{~mL}$ DMSO in a $250 \mathrm{~mL}$ round bottom flask. Then, $10 \mathrm{~g}$ PEG-diacid dissolved into $20 \mathrm{~mL}$ DMSO was added dropwise over 1 hour, and stirring of the reaction mixture was continued overnight $(24 \mathrm{~h})$. After evaporating off DMSO, the product mixture was collected and dialyzed $(\mathrm{MWCO}=1000)$ over 2 days. Finally, the PEG-DTP obtained by freeze-drying.

\subsection{Preparation of PEG-DTP/ADA Hydrogel}

PEG-DTP/ADA hydrogel was prepared by a simple procedure. The details are as follows. PEG-DTP and ADA were dissolved in PBS solution ( $\mathrm{pH}=7.4)$. Then, $0.25 \mathrm{~g}$ ADA aqueous solutions (20 wt \%) and $0.75 \mathrm{~g}$ PEG-DTP aqueous solutions (20 wt \%) were mixed at room temperature. Several minutes later, the mixed solutions gelled. The obtained $20 \mathrm{wt} \%$ hydrogel was named as $\mathrm{Gel}_{20}-3$. Using $\mathrm{Gel}_{20}-3$ (Table S1) as the sample, the experiments of injectability, sol-gel transition, in vitro cytotoxicity evaluation, and self-healing were carried out. Detailed components of other gels 
are presented in Table S1. $0.25 \mathrm{~g}$ ADA aqueous solution (20 wt \%) and $0.75 \mathrm{~g}$ DTP aqueous solutions $(15 \mathrm{mg} / \mathrm{mL}$ ) were mixed to form DTP/ADA hydrogel.

\subsection{Characterizations}

A Nicolet IS-10 spectrometer (Thermo Fisher, Waltham, MA, USA) was used to record the FT-IR spectra of ADA and ALG. The scan interval was $4000-600 \mathrm{~cm}^{-1}$, with 32 scans at a resolution of $2 \mathrm{~cm}^{-1}$.

A rheometer (TA Instruments, AR-G2, New Castle, DE, USA) with a platform of $4 \mathrm{~cm}$ diameter was used to measure hydrogels' rheological behavior. The storage moduli $G^{\prime}$ and loss moduli $G^{\prime \prime}$ were studied at a constant-strain (1\%) mode. The frequency range was $0.1-100 \mathrm{rad} \mathrm{s}^{-1}$. The self-healing properties of hydrogels were measured at a constant frequency of $6.283 \mathrm{rad} \mathrm{s}^{-1}$ by a strain step cycled between $1 \%$ and $1200 \%$ at $25{ }^{\circ} \mathrm{C}$.

The gel-sol-gel transition experiment was conducted by inversion. $\mathrm{HCl}(5 \mathrm{M})$ and triethylamine were used to trigger $\mathrm{pH}$ responsive gel-sol-gel transitions. First, $\mathrm{HCl}(10 \mu \mathrm{L}, 5 \mathrm{M})$ was added to the hydrogel. After $12 \mathrm{~h}$, equimolar triethylamine was added. For redox responsive gel-sol-gel transitions, we used DTT and $\mathrm{H}_{2} \mathrm{O}_{2}$ as the trigger. First, $16 \mathrm{mg}$ DTT was added into the $1 \mathrm{~g}$ hydrogel samples. After $12 \mathrm{~h}$, equivalent $\mathrm{H}_{2} \mathrm{O}_{2}(\sim 10 \mu \mathrm{L})$ was added and the hydrogels reformed.

The degradation properties of PEG-DTP/ADA hydrogels were determined by retention in different solutions. Hydrogels were submerged in PBS buffer ( $\mathrm{pH}=7.4$ and 5.0), $10 \mathrm{mM}(\mathrm{pH}=7.4)$ DTT solution, and $1 \mathrm{mM}$ DTT ( $\mathrm{pH}=7.4$ ) solution. The hydrogels were freeze-dried and weighed $\left(\mathrm{W}_{0}\right)$. Then, the hydrogels were immersed into PBS buffer $(20 \mathrm{~mL})$ or different concentrations of DTT solution $(20 \mathrm{~mL})$. After a certain period of time, the hydrogels were taken out, freeze dried, and weighed again $\left(\mathrm{W}_{\mathrm{t}}\right) . \mathrm{W}_{\mathrm{t}} / \mathrm{W}_{0} \times 100 \%$ was used to calculate the hydrogel's degradation ratio. The tests were conducted three times to confirm precision.

Rhodamine $\mathrm{B}$ was used as the model drug to analyze the drug release ability of hydrogel. Several $\mathrm{Gel}_{20}-3$ hydrogels loaded with rhodamine B were prepared as follows. First, PEG-DTP solutions $(0.75 \mathrm{~g} 20 \mathrm{wt} \%)$ and rhodamine B aqueous solution $(10 \mu \mathrm{L}, 1.0 \mathrm{mg} / \mathrm{mL})$ were mixed to obtain a drug solution. Then, the drug solution and ADA solution $(0.25 \mathrm{~mL} 20 \mathrm{wt} \%)$ were mixed to prepare drug-containing hydrogel samples. The four samples were incubated in $10 \mathrm{~mL}$ PBS $(\mathrm{pH}=7.4,5.0)$ buffer and $10 \mathrm{~mL}$ DTT $(10 \mathrm{mM}, \mathrm{pH}=7.4)$ solution or $10 \mathrm{~mL}$ DTT $(1 \mathrm{mM}, \mathrm{pH}=7.4)$ solution, respectively. At specific time points, sample solutions $(4 \mathrm{~mL})$ were withdrawn for UV-vis analysis and then the same volume of fresh solution was put back into the incubator. The $555 \mathrm{~nm}$ absorption of UV-vis were recorded to represent the concentration of rhodamine B. The tests were conducted three times. The cell compatibility of the designed hydrogels was studied by MTT assay. A549 cells cultured in 96-well plates at a density of $10^{4}$ cells per well were incubated at $37^{\circ} \mathrm{C}$ under $5 \%$ $\mathrm{CO}_{2}$. One hundred microliters of DMEM with $10 \%$ fetal bovine serum and $5 \%$ penicillin were added to each well. After $24 \mathrm{~h}$ incubation, the DMEM was replaced with a fresh culture medium, and then the prepared polymer ( $\mathrm{PEG}_{2 \mathrm{~K}}, \mathrm{ADA}$, and PEG-DTP) solutions (diluted to $1.5 \mathrm{wt} \%$ ) and hydrogel extract were added at a specific concentration. Then, the cells were incubated for $24 \mathrm{~h}$. The MTT solution ( $20 \mu \mathrm{L}, 5 \mathrm{mg} / \mathrm{mL}$ in PBS buffer) was added to each well and then cultured for another $4 \mathrm{~h}$. The medium in each well was removed and the purple formazan crystals from viable cells was dissolved by $200 \mu \mathrm{L}$ DMSO. The cell viability was analyzed by the absorbance at a wavelength of $480 \mathrm{~nm}$ by a micro-plate reader (Thermo Fisher, Waltham, MA, USA).

\section{Conclusions}

In this work, we used handily modifiable polysaccharide-oxidized sodium alginate and a macromolecular crosslinker, PEG-DTP, to prepare an injectable, dual responsive, and self-healing hydrogel via Schiff base crosslinking reaction. The stiffness and dehydration of oxidized sodium alginate at low $\mathrm{pH}$ caused the poor mechanical performance and $\mathrm{pH}$ responsiveness of the hydrogel. However, the introduction of PEG-DTP effectively improved the flexibility and hydrophilicity of 
hydrogel network and the ADA/PEG-DTP hydrogel exhibited better mechanical performance and a $\mathrm{pH}$-induced reversible sol-gel transition. With the dynamic acylhydrazone bonds and disulfide linkages, the ADA/PEG-DTP hydrogel showed dual responsive ( $\mathrm{pH}$ and redox) properties and the degradation rate or drug release of the hydrogel could be well controlled by varying the concentration of DTT solution or the $\mathrm{pH}$. Moreover, the hydrogels could self-repair nearly $100 \%$ after breakage because of the reversible dynamic acylhydrazone bonds. The in vitro cytotoxicity tests showed the hydrogel had excellent cytocompatibility. Therefore, the sodium alginate-based hydrogel integrated with multi-stimuli responsiveness, injectability, and self-healing ability showed potential for use in the fields of drug delivery, tissue engineering, and controlled 3D cell culture, etc.

Supplementary Materials: The following are available online. Figure S1: Schematic diagram of formation of ADA and PEG-DTP, Figure S2: FT-IR spectra of sodium alginate (ALG) and oxidized alginate (ADA), Figure S3: ${ }^{1} \mathrm{H}-\mathrm{NMR}$ of PEG-diacid in $\mathrm{CDCl}_{3}$, Figure $\mathrm{S} 4:{ }^{1} \mathrm{H}-\mathrm{NMR}$ of PEG-DTP in $\mathrm{CDCl}_{3}$, Figure S5: Photograph of PEG-DTP solution, ADA solution and hydrogel, Table S1: Preparation of PEG-DTP/ADA hydrogel with different contents, Table S2: Gelation time of $\mathrm{Gel}_{20}-3$ measured by vial inversion test.

Acknowledgments: This work was supported by the National Natural Science Foundation of China (No. 51273189), the National Science and Technology Major Project of the Ministry of Science and Technology of China (No. 2016ZX05016), and the National Science and Technology Major Project of the Ministry of Science and Technology of China (No. 2016ZX05046).

Author Contributions: Haiyang Yang and Lei Wang conceived and designed the experiments; Lei Wang performed the experiments; Chao Xu and Quan Tang analyzed the data; Wanfu Zhou and Qingguo Wang contributed reagents/materials/analysis tools; Lei Wang wrote the paper.

Conflicts of Interest: The authors declare no conflict of interest.

\section{References}

1. Zhang, Y.; Sun, Y.; Yang, X.; Hilborn, J.; Heerschap, A.; Ossipov, D.A. Injectable in situ forming hybrid iron oxide-hyaluronic acid hydrogel for magnetic resonance imaging and drug delivery. Macromol. Biosci. 2014, 14, 1249-1259. [CrossRef] [PubMed]

2. Mahanta, A.K.; Senapati, S.; Maiti, P. A polyurethane-chitosan brush as an injectable hydrogel for controlled drug delivery and tissue engineering. Polym. Chem. 2017, 8, 6233-6249. [CrossRef]

3. Zhu, Y.; Zhang, J.; Yang, J.; Pan, C.; Xu, T.; Zhang, L. Zwitterionic hydrogels promote skin wound healing. J. Mater. Chem. B 2016, 4, 5105-5111. [CrossRef]

4. Li, W.; Wang, J.; Ren, J.; Qu, X. 3D graphene oxide-polymer hydrogel: Near-infrared light-triggered active scaffold for reversible cell capture and on-demand release. Adv. Mater. 2013, 25, 6737-6743. [CrossRef] [PubMed]

5. Zhang, Y.; Fu, C.; Li, Y.; Wang, K.; Wang, X.; Wei, Y.; Tao, L. Synthesis of an injectable, self-healable and dual responsive hydrogel for drug delivery and 3D cell cultivation. Polym. Chem. 2017, 8, 537-544. [CrossRef]

6. Ravichandran, R.; Griffith, M.; Phopase, J. Applications of self-assembling peptide scaffolds in regenerative medicine: The way to the clinic. J. Mater. Chem. B 2014, 2, 8466-8478. [CrossRef]

7. Hall, D.J.; Urban, R.M.; Pourzal, R.; Turner, T.M.; Skipor, A.K.; Jacobs, J.J. Nanoscale surface modification by anodic oxidation increased bone ingrowth and reduced fibrous tissue in the porous coating of titanium-alloy femoral hip arthroplasty implants. J. Biomed. Mater. Res. B Appl. Biomater. 2017, 105, 283-290. [CrossRef] [PubMed]

8. Hoque, J.; Bhattacharjee, B.; Prakash, R.G.; Paramanandham, K.; Haldar, J. Dual function injectable hydrogel for controlled release of antibiotic and local antibacterial therapy. Biomacromolecules 2018, 19, 267-278. [CrossRef] [PubMed]

9. Hu, J.; Chen, Y.; Li, Y.; Zhou, Z.; Cheng, Y. A thermo-degradable hydrogel with light-tunable degradation and drug release. Biomaterials 2017, 112, 133-140. [CrossRef] [PubMed]

10. Zhou, T.; Jia, X.; Zhao, X.; Li, J.; Liu, P. Facile preparation of pH/reduction dual-responsive prodrug nanohydrogels for tumor-specific intracellular triggered release with enhanced anticancer efficiency. J. Mater. Chem. B 2017, 5, 2840-2848. [CrossRef] 
11. Gyarmati, B.; Vajna, B.; Nemethy, A.; Laszlo, K.; Szilagyi, A. Redox- and pH-responsive cysteamine-modified poly(aspartic acid) showing a reversible sol-gel transition. Macromol. Biosci. 2013, 13, 633-640. [CrossRef] [PubMed]

12. Chang, R.; An, H.; Li, X.; Zhou, R.; Qin, J.; Tian, Y.; Deng, K. Self-healable polymer gels with multi-responsiveness of gel-sol-gel transition and degradability. Polym. Chem. 2017, 8, 1263-1271. [CrossRef]

13. Du, Z.; Li, N.; Hua, Y.; Shi, Y.; Bao, C.; Zhang, H.; Yang, Y.; Lin, Q.; Zhu, L. Physiological pH-dependent gelation for $3 \mathrm{D}$ printing based on the phase separation of gelatin and oxidized dextran. Chem. Commun. 2017, 53, 13023-13026. [CrossRef] [PubMed]

14. Singh, N.K.; Lee, D.S. In situ gelling $\mathrm{pH}$ - and temperature-sensitive biodegradable block copolymer hydrogels for drug delivery. J. Control. Release 2014, 193, 214-227. [CrossRef] [PubMed]

15. Chen, J.; Ma, X.; Dong, Q.; Song, D.; Hargrove, D.; Vora, S.R.; Ma, A.W.K.; Lu, X.; Lei, Y. Self-healing of thermally-induced, biocompatible and biodegradable protein hydrogel. RSC Adv. 2016, 6, 56183-56192. [CrossRef]

16. Parlato, M.; Reichert, S.; Barney, N.; Murphy, W.L. Poly(ethylene glycol) hydrogels with adaptable mechanical and degradation properties for use in biomedical applications. Macromol. Biosci. 2014, 14, 687-698. [CrossRef] [PubMed]

17. Guo, R.; Su, Q.; Zhang, J.; Dong, A.; Lin, C.; Zhang, J. Facile access to multisensitive and self-healing hydrogels with reversible and dynamic boronic ester and disulfide linkages. Biomacromolecules 2017, 18, 1356-1364. [CrossRef] [PubMed]

18. Chang, R.; Wang, X.; Li, X.; An, H.; Qin, J. Self-activated healable hydrogels with reversible temperature responsiveness. ACS Appl. Mater. Interfaces 2016, 8, 25544-25551. [CrossRef] [PubMed]

19. Chen, Y.; Qian, W.; Chen, R.; Zhang, H.; Li, X.; Shi, D.; Dong, W.; Chen, M.; Zhao, Y. One-pot preparation of autonomously self-healable elastomeric hydrogel from boric acid and random copolymer bearing hydroxyl groups. ACS Macro Lett. 2017, 6, 1129-1133. [CrossRef]

20. Li, Z.; Lu, W.; Ngai, T.; Le, X.; Zheng, J.; Zhao, N.; Huang, Y.; Wen, X.; Zhang, J.; Chen, T. Mussel-inspired multifunctional supramolecular hydrogels with self-healing, shape memory and adhesive properties. Polym. Chem. 2016, 7, 5343-5346. [CrossRef]

21. Yang, W.; Wu, X.; Liu, F.; Dou, Y.; Hu, Z.; Hao, W. A fluorescent, self-healing and pH sensitive hydrogel rapidly fabricated from hpamam and oxidized alginate with injectability. RSC Adv. 2016, 6, 34254-34260. [CrossRef]

22. Wu, X.; He, C.; Wu, Y.; Chen, X. Synergistic therapeutic effects of schiff's base cross-linked injectable hydrogels for local co-delivery of metformin and 5-fluorouracil in a mouse colon carcinoma model. Biomaterials 2016, 75, 148-162. [CrossRef] [PubMed]

23. Yang, X.; Liu, G.; Peng, L.; Guo, J.; Tao, L.; Yuan, J.; Chang, C.; Wei, Y.; Zhang, L. Highly efficient self-healable and dual responsive cellulose-based hydrogels for controlled release and 3D cell culture. Adv. Funct. Mater. 2017, 27, 1703174. [CrossRef]

24. Halacheva, S.S.; Adlam, D.J.; Hendow, E.K.; Freemont, T.J.; Hoyland, J.; Saunders, B.R. Injectable biocompatible and biodegradable $\mathrm{pH}$-responsive hollow particle gels containing poly(acrylic acid): The effect of copolymer composition on gel properties. Biomacromolecules 2014, 15, 1814-1827. [CrossRef] [PubMed]

25. Yan, S.; Wang, T.; Feng, L.; Zhu, J.; Zhang, K.; Chen, X.; Cui, L.; Yin, J. Injectable in situ self-cross-linking hydrogels based on poly(L-glutamic acid) and alginate for cartilage tissue engineering. Biomacromolecules 2014, 15, 4495-4508. [CrossRef] [PubMed]

26. Clark, M.; Kiser, P. In situcrosslinked hydrogels formed using $\mathrm{Cu}(\mathrm{I})$-free huisgen cycloaddition reaction. Polym. Int. 2009, 58, 1190-1195. [CrossRef]

27. Niu, G.; Du, F.; Song, L.; Zhang, H.; Yang, J.; Cao, H.; Zheng, Y.; Yang, Z.; Wang, G.; Yang, H.; et al. Synthesis and characterization of reactive poloxamer 407s for biomedical applications. J. Control. Release 2009, 138, 49-56. [CrossRef] [PubMed]

28. Yuan, L.; Wu, Y.; Gu, Q.S.; El-Hamshary, H.; El-Newehy, M.; Mo, X. Injectable photo crosslinked enhanced double-network hydrogels from modified sodium alginate and gelatin. Int. J. Biol. Macromol. 2017, 96, 569-577. [CrossRef] [PubMed] 
29. Habibizadeh, M.; Rostamizadeh, K.; Dalali, N.; Ramazani, A. Preparation and characterization of pegylated multiwall carbon nanotubes as covalently conjugated and non-covalent drug carrier: A comparative study. Mater. Sci. Eng. C Mater. Biol. Appl. 2017, 74, 1-9. [CrossRef] [PubMed]

Sample Availability: Samples of the compounds are available from the authors.

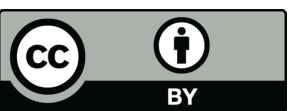

(C) 2018 by the authors. Licensee MDPI, Basel, Switzerland. This article is an open access article distributed under the terms and conditions of the Creative Commons Attribution (CC BY) license (http:/ / creativecommons.org/licenses/by/4.0/). 